\title{
Translational Stroke Research on Blood-Brain Barrier Damage: Challenges, Perspectives, and Goals
}

\author{
Yejie Shi ${ }^{1}$ - Rehana K. Leak ${ }^{2} \cdot$ Richard F. Keep ${ }^{3} \cdot$ Jun Chen $^{1}$
}

Received: 31 December 2015 / Accepted: 5 January 2016/Published online: 13 January 2016

(C) Springer Science+Business Media New York 2016

Over the past few decades, basic and clinical research has identified numerous risk factors for the development of stroke and led to major improvements in health management in the USA. As a result of these efforts, the relative rate of stroke death dropped by $33.7 \%$, and the actual occurrence of stroke deaths fell by $18.2 \%$ in the decade spanning from 2003 to 2013, according to the American Heart Association. Thus, stroke fell from the fourth to the fifth leading cause of death in 2013, behind heart disease, cancer, chronic lower respiratory diseases, and unintentional injuries. These improvements are largely attributed to superior control of hypertension, diabetes mellitus, high cholesterol, and tobacco use [1]. To date, the treatment of acute ischemic stroke is largely dependent on recanalization using recombinant tissue-type plasminogen activator (tPA) in the appropriate patient population $[2,3]$. Encouragingly, recent clinical trials have demonstrated significant benefits for intra-arterial thrombectomy in a subset of acute stroke patients with intracranial large artery occlusion [4]. Despite these improvements in population health and stroke treatment, stroke still remains a leading cause of longterm disability and approximately 795,000 people experience a new or recurrent stroke every year [1]. Thus, basic and

Jun Chen

chenj2@upmc.edu

1 Center of Cerebrovascular Disease Research, University of Pittsburgh School of Medicine, S-507 Biomedical Science Tower 3500 Terrace Street, Pittsburgh, PA 15213, USA

2 Division of Pharmaceutical Sciences, Mylan School of Pharmacy, Duquesne University, Pittsburgh, PA 15282, USA

3 Department of Neurosurgery, University of Michigan, Ann Arbor, MI 48109, USA clinical investigations of the mechanisms underlying ischemic brain injury must remain an urgent priority in order to promote the discovery of novel therapeutic targets and improve the safety and efficacy of current tPA and thrombectomy treatments.

During and after ischemic stroke, loss of blood-brain barrier (BBB) integrity is a prominent pathological event that contributes to further evolution of the injury. BBB dysfunction is also a hallmark of intracerebral hemorrhage [5, 6]. Despite its obvious clinical relevance, $\mathrm{BBB}$ protection has received much less attention than is warranted. An impaired BBB not only facilitates the development of brain edema and neuroinflammation, but also increases the risk of lethal hemorrhagic transformation during thrombolysis, thereby limiting the use of tPA and leading to poor patient outcomes [7, 8]. Recent advances in stroke telemedicine provide an effective and promising method to increase the use of tPA therapy [9], which, together with the growing application of thrombectomy, is likely to improve post-ischemia reperfusion in a larger population of stroke patients in the near future. As this treatment method works better when the BBB remains intact, therapeutic strategies aimed at neurovascular unit protection and prevention of BBB damage after ischemia/reperfusion (I/R) need to be better prioritized in stroke research.

In earlier reports, it was widely held that all forms of BBB rupture after I/R were the direct consequence of matrix metalloproteinase (MMP)-mediated degradation of endothelial intercellular junctions and basal lamina [10-14]. However, recent animal models of stroke have revealed a complex, biphasic temporal profile of BBB breakdown, with an immediate phase of early BBB hyperpermeability $4-6 \mathrm{~h}$ after stroke, followed by a delayed opening of the BBB 48-72 $\mathrm{h}$ after stroke. In recent years, the availability of advanced imaging techniques and novel transgenic animal models have greatly facilitated research on BBB dysfunction after stroke, with 
an increasing focus on the initiation of the early breach at the level of the endothelial cell. Unexpectedly, studies have shown that the degradation of tight junctional proteinsgenerally held responsible for BBB opening after strokeoccurs much later than the pathological hyperpermeability of the BBB [15]. Enhanced transendothelial vesicle trafficking has been reported instead, which may represent an important mechanism neglected in older studies [16, 17]. A recent study revealed additional, subtle microarchitectural changes in the cytoskeleton of brain microvascular endothelial cells, which occur well before the frank degradation of endothelial junctions and basement membrane by the MMPs [18]. For example, I/R-enhanced actin polymerization in endothelial cells is a driving force for increased paracellular permeability and renders the BBB much more susceptible to further attack by infiltrating peripheral immune components [18]. The actin cytoskeleton and tight junctions are physically linked and cytoskeletal changes may impact BBB function without the overt loss of tight junction proteins $[18,19]$. Together, these studies have lent greater insight into early pathophysiological changes in the BBB immediately after $\mathrm{I} / \mathrm{R}$ and shed new light on components of this injury. Endothelial celltargeted interventions have also begun to tackle a fundamentally important question: is early impairment of the $\mathrm{BBB}$ one of the causes, rather than only a consequence of brain parenchymal injury in stroke? In this scenario, BBB destruction and parenchymal injury likely exacerbate each other in a feed-forward or self-amplifying spiral. If this hypothesis continues to garner support from further studies, maintenance of the BBB immediately after stroke onset might be expected to brake the downstream progression of ischemic brain injury, improve neurological outcomes, and perhaps even save lives.

In addition to the actin cytoskeleton, emerging evidence also supports a critical role for other BBB cell components, such as astrocytes and pericytes, in the regulation of BBB stability [20-22]. Besides their participation in the development and maintenance of normal BBB integrity [23], these cells are thought to contribute to postinjury BBB repair. Brain vascular pericytes, for example, appear to acquire multipotent stem cell activity following ischemia and become capable of differentiating into cells of neural or vascular lineage, thereby reconstructing the neurovascular unit [24]. Furthermore, astrocytes possess end-feet that envelop brain capillaries and become swollen after stroke injury, perhaps serving to limit the entry of plasma factors and blood into the parenchyma of the brain [25]. Other cells in the neurovascular unit, including oligodendrocyte precursor cells [26] and microglia [27], also influence BBB function via secreted factors and are likely to engage in crosstalk [28]. How different cell types and cell type-specific mechanisms contribute to BBB dysfunction remains poorly studied and warrants deeper investigation; cell-specific gene-targeting strategies are especially powerful in this regard. The choice of appropriate tracers to assess the permeability of the BBB also cannot be understated, as different tracers use distinct pathways to diffuse across the BBB, through paraendothelial or trans-endothelial mechanisms $[16,29,30]$. There also remains a need to develop advanced molecular imaging techniques to detect BBB penetration of tracers during early stages of $\mathrm{I} / \mathrm{R}$. This might enable early detection of BBB dysfunction in the clinical setting, which would offer the distinct advantage of targeting thrombolysis to the appropriate patient population. Furthermore, we need to improve our understanding of the context dependency of the mechanisms underlying BBB breakdown, as there might be unique changes in different cell types at various stages of injury development.

As the major point of separation of brain parenchyma from circulating blood, the BBB also serves as a dynamic neuroimmune interface where multicellular interactions transpire. The neuroinflammatory responses to $\mathrm{I} / \mathrm{R}$ include the following: (1) parenchymal inflammation, which is primarily attributed to I/R-activated endothelial cells, (2) subsequent release of various pro-inflammatory meditators such as cytokines and chemokines, and (3) peripheral immune changes such as leukocyteendothelial interactions via selectins, adhesion molecules, and chemokines/chemokine receptors [31]. Whereas the severely injured BBB freely permits the infiltration of peripheral immune cells, blocking early BBB damage ameliorates secondary injuries caused by proinflammatory responses, blocks a self-amplifying cascade of tissue destruction, and offers persistent histological and functional protection [18]. Aside from deleterious actions, some immune responses also actively promote the reestablishment of BBB integrity following injury, as might be expected from the primary role of the immune system in self-repair and healing [32, 33]. Finetuning these immune responses to achieve homeostasis at the neuroimmune interface is an important research topic for the restoration of BBB function.

There are several important directions for future preclinical studies on the BBB. First, employment of translatable model systems is essential, e.g., use of an embolic stroke model that encompasses tPA-induced reperfusion $[34,35]$. Future studies are warranted to determine whether early BBB impairment might predict the risk of hemorrhagic transformation upon tPA recanalization and influence stroke prognosis. Second, we need to target our treatments to different cell types at distinct stages of injury, because different mechanisms may dominate specific stages of stroke injury. Third, the pathophysiology of BBB disruption must be evaluated 
in the context of age, gender, and comorbid conditions such as hypertension, hyperglycemia, and high cholesterol $[1,36,37]$. These variables likely promote the clinically heterogeneous profiles of stroke. Finally, attention should be given not only to acute protection against BBB damage but also to structural and functional restoration of the BBB, such as repair of tight junctions, in order to ensure a profound and sustained improvement in long-term neurological outcomes. Such approaches might also reduce the risk of stroke recurrence where there may be proinflammatory changes in the cerebral endothelium.

In conclusion, a fruitful collaboration between scientific researchers and the medical establishment is partly responsible for recent improvements in health outcomes and significant reductions in mortality after stroke. A new research emphasis on restoring the neurovascular unit now raises hope of long-lasting protection of the BBB after stroke [38]. If parenchymal injury lies downstream of early BBB damage, or even if they only exacerbate each other in a feed-forward loop, finding the therapeutic means to restore BBB integrity is expected to pay large dividends, given the current use of thrombolysis and interventional recanalization and the risk for hemorrhagic transformation. Success in this arena may provide the necessary momentum for continued improvements in stroke outcomes over the next few decades so that the recent positive trajectories in mortality reduction do not flag.

Acknowledgments Y.S. is a recipient of the American Heart Association Fellowship 15POST22260011. This work was supported by the U.S. National Institutes of Health grants NS089534 and NS045048 (to J.C.), and the U.S. Department of Veterans Affairs (VA) Merit Review Award BX002495 (to J.C.). J.C. was supported by the VA Research Career Scientist Award.

\section{Compliance with Ethical Standards}

Conflict of Interest The authors declare that they have no competing interests.

\section{References}

1. Mozaffarian D, Benjamin EJ, Go AS, Arnett DK, Blaha MJ, Cushman M et al. Heart Disease and Stroke Statistics-2016 Update: A Report From the American Heart Association. Circulation. 2015.

2. Powers WJ, Derdeyn CP, Biller J, Coffey CS, Hoh BL, Jauch EC, et al. American heart association/american stroke association focused update of the 2013 guidelines for the early management of patients with acute ischemic stroke regarding endovascular treatment: a guideline for healthcare professionals from the American Heart Association/American Stroke Association. Stroke. 2015;46(10):3020-35.

3. Lapchak PA. Critical early thrombolytic and endovascular reperfusion therapy for acute ischemic stroke victims: a call for adjunct neuroprotection. Transl Stroke Res. 2015;6(5):345-54.
4. Grotta JC, Hacke W. Stroke neurologist's perspective on the new endovascular trials. Stroke. 2015;46(6):1447-52.

5. Keep RF, Xiang J, Ennis SR, Andjelkovic A, Hua Y, Xi G, et al. Blood-brain barrier function in intracerebral hemorrhage. Acta Neurochir Suppl. 2008;105:73-7.

6. Keep RF, Zhou N, Xiang J, Andjelkovic AV, Hua Y, Xi G. Vascular disruption and blood-brain barrier dysfunction in intracerebral hemorrhage. Fluids Barriers CNS. 2014;11:18.

7. Cheng Y, Xi G, Jin H, Keep RF, Feng J, Hua Y. Thrombin-induced cerebral hemorrhage: role of protease-activated receptor-1. Transl Stroke Res. 2014;5(4):472-5.

8. Khanna A, Kahle KT, Walcott BP, Gerzanich V, Simard JM. Disruption of ion homeostasis in the neurogliovascular unit underlies the pathogenesis of ischemic cerebral edema. Transl Stroke Res. 2014;5(1):3-16.

9. Audebert HJ, Kukla C, Von Clarmann Claranau S, Kuhn J, Vatankhah B, Schenkel J, et al. Telemedicine for safe and extended use of thrombolysis in stroke: the telemedic pilot project for integrative stroke care (TEMPiS) in Bavaria. Stroke. 2005;36(2):28791.

10. Asahi M, Wang X, Mori T, Sumii T, Jung JC, Moskowitz MA, et al. Effects of matrix metalloproteinase-9 gene knock-out on the proteolysis of blood-brain barrier and white matter components after cerebral ischemia. J Neurosci. 2001;21(19):7724-32.

11. Liu J, Jin X, Liu KJ, Liu W. Matrix metalloproteinase-2-mediated occludin degradation and caveolin-1-mediated claudin-5 redistribution contribute to blood-brain barrier damage in early ischemic stroke stage. J Neurosci. 2012;32(9):3044-57.

12. Yang Y, Estrada EY, Thompson JF, Liu W, Rosenberg GA. Matrix metalloproteinase-mediated disruption of tight junction proteins in cerebral vessels is reversed by synthetic matrix metalloproteinase inhibitor in focal ischemia in rat. J Cereb Blood Flow Metab. 2007;27(4):697-709.

13. Reuter B, Rodemer C, Grudzenski S, Meairs S, Bugert P, Hennerici $\mathrm{MG}$, et al. Effect of simvastatin on MMPs and TIMPs in human brain endothelial cells and experimental stroke. Transl Stroke Res. 2015;6(2):156-9.

14. Neuwelt EA, Bauer B, Fahlke C, Fricker G, Iadecola C, Janigro D, et al. Engaging neuroscience to advance translational research in brain barrier biology. Nat Rev Neurosci. 2011;12(3):169-82.

15. Krueger M, Hartig W, Reichenbach A, Bechmann I, Michalski D. Blood-brain barrier breakdown after embolic stroke in rats occurs without ultrastructural evidence for disrupting tight junctions. PLoS One. 2013;8(2), e56419.

16. Knowland D, Arac A, Sekiguchi KJ, Hsu M, Lutz SE, Perrino J, et al. Stepwise recruitment of transcellular and paracellular pathways underlies blood-brain barrier breakdown in stroke. Neuron. 2014;82(3):603-17.

17. Nahirney PC, Reeson P, Brown CE. Ultrastructural analysis of blood-brain barrier breakdown in the peri-infarct zone in young adult and aged mice. J Cereb Blood Flow Metab. 2015.

18. Shi Y, Zhang L, Pu H, Mao L, Hu X, Jiang X et al. Rapid endothelial cytoskeletal reorganization enables early blood-brain barrier disruption and long-term ischaemic reperfusion brain injury. Nat Commun. 2016;7:10523. doi: 10.1038/ncomms 10523

19. Lin T, Zeng L, Liu Y, DeFea K, Schwartz MA, Chien S, et al. RhoROCK-LIMK-cofilin pathway regulates shear stress activation of sterol regulatory element binding proteins. Circ Res. 2003;92(12): 1296-304.

20. Armulik A, Genove G, Mae M, Nisancioglu MH, Wallgard E, Niaudet $\mathrm{C}$, et al. Pericytes regulate the blood-brain barrier. Nature. 2010;468(7323):557-61.

21. Daneman R, Zhou L, Kebede AA, Barres BA. Pericytes are required for blood-brain barrier integrity during embryogenesis. Nature. 2010;468(7323):562-6. 
22. Yao Y, Chen ZL, Norris EH, Strickland S. Astrocytic laminin regulates pericyte differentiation and maintains blood brain barrier integrity. Nat Commun. 2014;5:3413.

23. Hall CN, Reynell C, Gesslein B, Hamilton NB, Mishra A, Sutherland BA, et al. Capillary pericytes regulate cerebral blood flow in health and disease. Nature. 2014;508(7494): 55-60.

24. Nakagomi T, Kubo S, Nakano-Doi A, Sakuma R, Lu S, Narita A, et al. Brain vascular pericytes following ischemia have multipotential stem cell activity to differentiate into neural and vascular lineage cells. Stem Cells. 2015;33(6):1962-74.

25. Xiang J, Tang Y, Li C, Su EJ, Lawrence DA, Keep RF. Mechanisms underlying astrocyte endfeet swelling in stroke. Acta Neurochir Suppl. 2016;121:19-22.

26. Seo JH, Maki T, Maeda M, Miyamoto N, Liang AC, Hayakawa K, et al. Oligodendrocyte precursor cells support blood-brain barrier integrity via TGF-beta signaling. PLoS One. 2014;9(7), e103174.

27. da Fonseca AC, Matias D, Garcia C, Amaral R, Geraldo LH, Freitas $\mathrm{C}$, et al. The impact of microglial activation on blood-brain barrier in brain diseases. Front Cell Neurosci. 2014;8:362.

28. Egashira Y, Hua Y, Keep RF, Xi G. Intercellular cross-talk in intracerebral hemorrhage. Brain Res. 2015;1623:97-109.

29. Li H, Gao A, Feng D, Wang Y, Zhang L, Cui Y, et al. Evaluation of the protective potential of brain microvascular endothelial cell autophagy on blood-brain barrier integrity during experimental cerebral ischemia-reperfusion injury. Transl Stroke Res. 2014;5(5):61826.

30. Merali Z, Leung J, Mikulis D, Silver F, Kassner A. Longitudinal assessment of imatinib's effect on the blood-brain barrier after ischemia/reperfusion injury with permeability MRI. Transl Stroke Res. 2015;6(1):39-49.

31. Iadecola C, Anrather J. The immunology of stroke: from mechanisms to translation. Nat Med. 2011;17(7):796-808.

32. Gliem M, Krammes K, Liaw L, van Rooijen N, Hartung HP, Jander $\mathrm{S}$. Macrophage-derived osteopontin induces reactive astrocyte polarization and promotes re-establishment of the blood brain barrier after ischemic stroke. Glia. 2015;63(12):2198-207.

33. Yang Y, Salayandia VM, Thompson JF, Yang LY, Estrada EY, Yang Y. Attenuation of acute stroke injury in rat brain by minocycline promotes blood-brain barrier remodeling and alternative microglia/ macrophage activation during recovery. J Neuroinflammation. 2015;12:26.

34. Hoda MN, Bhatia K, Hafez SS, Johnson MH, Siddiqui S, Ergul A, et al. Remote ischemic perconditioning is effective after embolic stroke in ovariectomized female mice. Transl Stroke Res. 2014;5(4):484-90.

35. Zhu W, Libal NL, Casper A, Bodhankar S, Offner H, Alkayed NJ. Recombinant $\mathrm{T}$ cell receptor ligand treatment improves neurological outcome in the presence of tissue plasminogen activator in experimental ischemic stroke. Transl Stroke Res. 2014;5(5):612-7.

36. Hafez S, Coucha M, Bruno A, Fagan SC, Ergul A. Hyperglycemia, acute ischemic stroke, and thrombolytic therapy. Transl Stroke Res. 2014;5(4):442-53.

37. Zhou Y, Murugappan SK, Sharma VK. Effect of clot aging and cholesterol content on ultrasound-assisted thrombolysis. Transl Stroke Res. 2014;5(5):627-34.

38. Zhang JH, Badaut J, Tang J, Obenaus A, Hartman R, Pearce WJ. The vascular neural network - a new paradigm in stroke pathophysiology. Nat Rev Neurol. 2012;8(12):711-6. 